PROCEEDINGS OF THE

AMERICAN MATHEMATICAL SOCIETY

Volume 127, Number 10, Pages 2961-2968

S 0002-9939(99)04907-2

Article electronically published on June 17, 1999

\title{
MIDCONVEX FUNCTIONS IN LOCALLY COMPACT GROUPS
}

\author{
A. CHADEMAN AND F. MIRZAPOUR
}

(Communicated by J. Marshall Ash)

\begin{abstract}
The theorems of Bernstein-Doetsch, and Ostrowski, concerning the continuity of midconvex functions are extended to open subsets of locally compact and root-approximable topological groups.
\end{abstract}

\section{INTRODUCTION}

In his original article [11], Jensen proved that a midconvex function $f$ on an open interval $\Omega=(\alpha, \beta)$ of the real line $\mathbf{R}$ is continuous at a point $a \in \Omega$, provided it satisfies $\limsup _{x \rightarrow a} f(x)<+\infty$. Furthermore he showed that $f$ is continuous providing it has finite limsup at each point, and especially if it is assumed to be upper semi-continuous or to be bounded from above. Many authors have been then interested in the problem of continuity of functions satisfying Jensen's functional inequality

$$
2 f(x) \leq f(x+h)+f(x-h)
$$

where $f: \Omega \rightarrow \mathbf{R}$ is a real function defined on a convex subset $\Omega$ of a real vector space $X$ on which a suitable topology is given, and the variables $x$ and $h$ are restricted only to the conditions $x, x+h, x-h \in \Omega$. These functions are also called convex in [11], convex, convex $(\mathrm{J})$, J-convex or midconvex in the literature. Wellknown classical theorems guarantee the continuity of $f$, provided it is assumed to be bounded from above on some rich subset of $\Omega$. The word rich is essentially interpreted here by topological or measure theoretical arguments. Very often $\Omega$ is also assumed to be open. We refer to the books $[14,18]$, and the references therein, for historical notes and to the articles $[12,13]$ and their references for more recent developments. A comprehesive treatment of the subject for the case $X=\mathbf{R}^{n}$ may be found in Kuczma [14]. Notable among the recent generalizations is the article of Kominek and Kuczma [13]. The generalization consists, essentially, in the fact that the topology on the space $X$ is semilinear. In the article of $\mathrm{Ng}$ and Nikodem [16] the Bernstein-Doetsch theorem is extended to approximately midconvex functions in a semilinear topological space. For extensions of these continuity theorems to other classes of functions, such as $n$-convex functions, one is referred to Ger $[7,8]$.

Concerning midconvex functions in topological groups, the theorems of Jensen and Blumberg-Sierpinski have been already proved in [6]. As this reference does not

Received by the editors December 12, 1996 and, in revised form, January 1, 1998.

1991 Mathematics Subject Classification. Primary 26A51; Secondary 22A10.

Key words and phrases. Midconvex functions, locally compact groups, Bernstein-Doetsch theorem, Jensen's theorem, Ostrowski's theorem. 
seem widely accessible, we shall recall, with some additional remarks, the definitions and the principal statements from [6] in the Preliminary section.

The object of the present paper is to extend the continuity theorems of BernsteinDoetsch, and Ostrowski by relaxing the convexity of $\Omega$ and assuming that $x+h$ and $x-h$ remain in a small neighborhood of $x$ only. In such a setting, the underlying space $X$ does not have to have a vector space structure, but only the structure of a topological group or semitopological group, or even a semitopological semigroup will suffice. However, we shall restrict ourselves here to the case of non discrete topological groups. This restriction will be respected in the entire paper without being expressly mentioned.

Jensen's theorem about upper semicontinuity remains valid (see the remark following the definition (5)), while his theorem about boundedness from above is no more valid for locally midconvex functions. This is shown by an elementary example consisting of the characteristic function of an open interval in $\mathbf{X}=R$. This example seems interesting, because all discontinuous midconvex functions, in the usual sense, are non measurable. Other conditions are needed to imply the continuity of such a locally midconvex function. We are led to introduce, as is done in [6], with slight modifications, the notions of locally and globally midconvex functions, as well as some intermediate ones, such as sequentially midconvex functions and locally uniformly midconvex functions. The usual midconvex functions, as used in [18, Chapter 7], corresponds to our globally midconvex ones. Some of the continuity theorems may be proved in any topological group, while for others the existence of successive square roots with an approximation property is needed. These groups are called root-approximable topological groups.

To be more precise, let $G$ be a (non-discrete) topological group with multiplicative law and let $\Phi$ denote the filter of neighborhoods of its neutral element $e$. Now we consider an open set $\Omega \subset G$, and a function $f: \Omega \longrightarrow[-\infty,+\infty]$. To avoid ambiguities, we do not allow $f$ take respectively $-\infty,+\infty$ at any two symmetric points $a y, a y^{-1} \in \Omega$ with respect to a point $a \in \Omega$. Whenever $G$ is locally compact, we suppose that it is endowed with a left invariant Haar measure $\mu>0$.

The organization of the article is as follows: First we give the definitions in the Preliminary section. Then we recall briefly finiteness and measurability results following [6]. The root-approximability and the Doetsch-Bernstein theorem will then follow. Finally midconvex hull and Ostrowski's theorem will be treated in the last section. Our main results here are the following theorems:

Theorem 1. A globally midconvex function in a midconvex open subset of a rootapproximable topological group which is bounded from above in some neighborhood of a point is everywhere continuous.

This is a generalization of the theorem of Bernstein-Doetsch [1].

Theorem 2. Let $\Omega$ be a midconvex open subset in a root-approximable locally compact abelian group $G$ endowed with a Haar measure $\mu$, and let $f: \Omega \longrightarrow \mathbf{R}$ be a globally midconvex function. If $f$ is bounded from above on a set $E \subset \Omega$ with $\mu(E)>0$, then $f$ must be continuous on $\Omega$.

This is a generalization of the theorem of Ostrowski [17]. 


\section{Preliminaries}

With the notations and conventions described in the introduction, here we shall give some definitions, and recall some known facts about finiteness and measurability of midconvex functions.

2.1. Definitions. 1) The function $f$ is called globally midconvex in $\Omega$ if for every $a, y$ such that $a, a y, a y^{-1} \in \Omega$, the midconvex inequality

$$
2 f(a) \leq f(a y)+f\left(a y^{-1}\right)
$$

holds.

2) Similarly, $f$ is said to be locally midconvex in $\Omega$ if for every $a \in \Omega$ there exists an open symmetric $V=V^{-1} \in \Phi$ such that the midconvex inequality (2) holds for every $y \in V$.

3) As an intermediate notion, we define $f$ to be sequentially midconvex at $a$, if it is locally midconvex in $\Omega$ and if there exists a symmetric open $V \in \Phi$ such that $a V^{2} \subset \Omega$, and if for each $y \in V$, the following inequality holds:

$$
2 f(a y) \leq f(a)+f\left(a y^{2}\right) .
$$

A function which is sequentially midconvex at each point of $\Omega$ is called sequentially midconvex in $\Omega$.

4) We say that $f$ is locally uniformly midconvex at a point $a$, if there exists a neighborhood $a V, V=V^{-1} \in \Phi$, such that $a V^{2} \subset \Omega$, and for each $y \in V$, the following condition is satisfied:

$$
\forall z \in V, 2 f(a y) \leq f\left(a y z^{-1}\right)+f(a y z) .
$$

5) Finally, let us explain how the classical notion of convex functions, defined in an open subset $\Omega$ of a topological vector space, is related to the above notion of locally midconvex functions in $\Omega$, considered as an open subset of the underlying topological group.

Let $X$ be a real topological vector space and $\Omega \subset X$ be open. A function $f$ with values in $[-\infty,+\infty]$ defined in $\Omega$ is convex in the usual sense if, and only if, it is locally midconvex, upper semicontinuous, and satisfies $f(x)<+\infty$ for all $x \in \Omega$.

By analogy, an upper semicontinuous and locally midconvex function with values in $[-\infty,+\infty)$, defined on an open subset $\Omega$ of a topological group, may be called convex as is done in [5].

Remark. Writing (2) in the form

$$
2 f(a)-f(a y) \leq f\left(a y^{-1}\right)
$$

and taking liminf in it, it is easily shown that a convex function is continuous ([5]). But the characteristic function $\chi_{A}$, where $A$ is any open subset with nonempty boundary is a locally midconvex function on $\Omega=G$ which is lower semicontinuous, discontinuous, and bounded. Taking $G=\mathbf{R}$ and $A=(0,1)$, one obtains a very elementary discontinuous example.

6) A group $G$ endowed with a topology $\mathcal{T}$ is said to be a root-approximable group if each $x \in G$ has a sequence of successive square roots converging to $e$. In other words to each $x \in G$ corresponds a sequence $\left(y_{n}\right)_{n \geq 0}$ of $2^{n}$-th roots of $x$ such that

$$
\lim _{n \rightarrow \infty} y_{n}=e, \quad y_{n}{ }^{2^{n}}=x,(n=0,1,2, \ldots) .
$$


In this definition, $(G, \mathcal{T})$ is not necessarily a topological group. By a root-approximable topological group we mean a topological group which is also root-approximable.

7) Let $G$ be a root-approximable topological group. For any subset $E \subset G$, we define by induction a sequence of subsets $H_{j}(E)$ in the following way: Let $H_{0}(E)=E$, and $H_{j}(E)$ be the midpoints set of $H_{j-1}(E)$, where by the midpoints set of a set $F$, we mean the set of all elements of $G$ of the form $x a$, provided that $x \in F, x a^{2} \in F$. The midconvex hull of $E$ is then by definition the union of all $H_{j}(E)$, for $0 \leq j<\infty$.

8) A subset $E$ of the topological group $G$ is called right midconvex if for every $x, y \in E$, there exists a $z \in G$ such that $x z \in E$ and $x z^{2}=y$.

Let us recall now some results from [6], concerning the finiteness and measurability of locally midconvex functions.

2.2. Finiteness. Being concerned with the continuity problem, under some additional hypothesis, we can restrict ourselves in the case of a locally compact group to finite valued locally midconvex functions. Let us observe first that we can find quite well pathological locally midconvex functions on $\Omega=G=\mathbf{R}$ such as

$$
f_{1}=\chi_{A} \times(+\infty), \quad f_{2}=\left(1-\chi_{A}\right) \times(-\infty),
$$

where $A=(0,1)$ and $\chi_{A}$ denotes the characteristic function of $A$ (notice that $f_{1}+f_{2}$ is not locally midconvex). More precisely, we have the following known results ([10, Chapter 4, Theorem 9.1b], [6, 18]):

Proposition 1. If $G$ is locally compact, then any locally midconvex function $f$ : $\Omega \longrightarrow[-\infty,+\infty]$, satisfying $f(x)<+\infty$, for $\mu$-almost every $x \in \Omega$, will satisfy $f(x)<+\infty$, for all $x \in \Omega$.

Remark. If $f: \Omega \longrightarrow[-\infty,+\infty]$ is locally midconvex, then $f^{-1}(-\infty)$ is always closed but not open in general. However, it follows from the definitions that in the case of a sequentially midconvex function $f$, this set is open as well and $f$ is continuous on it. More precisely, the following obvious ressult is much stronger than the continuity theorem of sequentially midconvex functions on their set of $-\infty$ ([6], Proposition 2).

Proposition 2. If $f: \Omega \longrightarrow[-\infty,+\infty]$ is sequentially midconvex at a point $a \in \Omega$, and if $f(a)=-\infty$, then $f$ is the constant $-\infty$ near $a$.

Remark. The sequential midconvexity cannot be replaced by local midconvexity in the above proposition. The function $f_{2}$ is a counter-example.

Concerning the continuity of sequentially midconvex functions, we may then restrict ourselves to real valued functions in open subsets of $G$. The precise theorem of Jensen may be generalized as follows:

Proposition 3 (Jensen's theorem). Suppose that $a \in \Omega, f: \Omega \longrightarrow \mathbf{R}$ is sequentially midconvex at a. If

$$
\limsup _{x \rightarrow e} f(a x)<+\infty
$$

then $f$ is continuous at a.

Corollary. A sequentially midconvex function $f: \Omega \longrightarrow \mathbf{R}$ is continuous if and only if it is locally bounded from above. 
2.3. Measurability. The continuity of measurable usual midconvex functions, in the classical setting of finite dimensional Euclidean spaces, proved by Blumberg [2] and Sierpinski [19] is one of the most striking old results. It tells us that midconvex functions are very regular or very irregular. As observed above, the situation is not the same for the local point of view. We have the following important theorem of Blumberg-Sierpinski:

Proposition 4. Suppose that $a \in \Omega$, and $f: \Omega \longrightarrow \mathbf{R}$ is locally uniformly midconvex at $a$. If $f$ is measurable, then $f$ is continuous at $a$.

Sketch of the proof (following [6]). Let $W=W^{-1} \in \Phi$ be open with compact closure $\bar{W}$. The modular function $\Delta$ has a positive minimum $m>0$ on $\bar{W}$, and satisfies

$$
\mu(A z)=\Delta(z) \mu(A)
$$

for every $z \in G$, and $\mu$-measurable set $A \subset G$ (see [20], p.39). One can choose (see Bourbaki [3]) $U, V \in \Phi$, such that $U^{2} \subset V, V^{2} \subset W$. The inequality (3) may be used. If $f$ is not bounded from above near $a$, then for each integer $n \geq 1$, there exists a $y_{n} \in U$ such that $f\left(a y_{n}\right)>n$. Define $\varphi_{n}: U \longrightarrow V$ by putting $\varphi_{n}(x)=x^{-1} y_{n}$. If $V$ is assumed to be sufficiently small, then the inequality (4) is satisfied. We use this assumption throughout the proof. Now given an arbitrary $x \in U$, let $z_{n}=\varphi_{n}(x)$, and substitute $y_{n}$ and $z_{n}$ for $y$ and $z$ in the above inequality. We obtain:

$$
2 f\left(a y_{n}\right) \leq f(a x)+f\left(a y_{n} \varphi_{n}(x)\right) .
$$

Next we define, for each $n \geq 1$,

$$
A_{n}=\{x \in U \mid f(a x)>n\}, \quad B_{n}=\{x \in U \mid f(a x) \leq n\} .
$$

It can be shown that the measure of $A_{n}$ is bounded from below by a positive constant independent of $n$ :

$$
\mu\left(A_{n}\right) \geq \frac{\alpha m^{2}}{1+m^{2}}
$$

(with $\alpha=\mu(U))$. The sequence $\left(A_{n}\right)_{n \geq 1}$ satisfies

$$
\begin{gathered}
\mu\left(A_{1}\right) \leq \beta=\mu(W), \\
\forall n \geq 1, \quad A_{n+1} \subset A_{n} .
\end{gathered}
$$

The classical argument may be applied to obtain $\mu\left(\bigcap_{n>1} A_{n}\right) \geq m \alpha^{2} /\left(1+m^{2}\right)>0$. Hence, $A_{n}$ is not empty, which contradicts the standard analysis of real numbers.

Corollary 1. A real valued locally uniformly midconvex function in an open subset of any locally compact group is convex if and only if it is measurable with respect to the left-invariant Haar measure.

Corollary 2 (Blumberg-Sierpinski's Theorem for Topological Groups). If a real valued globally midconvex function in an open subset of a locally compact group is measurable with respect to its Haar measure, then it is continuous. 


\section{Root-Approximability AND the Bernstein-Doetsch theOREM}

To generalize the Bernstein-Doetsch theorem, the notion of root-approximable groups is introduced. There are interesting examples and counter-examples among the classical groups, left to the readers. The following lemma gives a convexity inequality with rational coefficients. The proof is done by induction.

Lemma 1. Suppose that $f: \Omega \longrightarrow \mathbf{R}$ is globally midconvex. Let $x, a \in G$ and $n \in \mathbf{N}$ be such that $\left\{x, x a, x a^{2}, \cdots, x a^{n}\right\} \subset \Omega$. Then for every integer $m \leq n$, we have

$$
f\left(x a^{m}\right) \leq(1-m / n) f(x)+(m / n) f\left(x a^{n}\right) .
$$

Theorem 1. Let $G$ be a root-approximable topological group, and $\Omega \subset G$ midconvex. Suppose that $f: \Omega \longrightarrow \mathbf{R}$ is globally midconvex. If there exist a point $a \in \Omega$ and a neighborhood $a V$ of $a, V \in \Phi$, such that $f$ is bounded from above on $a V$, then $f$ must be continuous in $\Omega$.

Proof. Suppose, without loss of generality, that $e \in \Omega$, and $f$ satisfies the inequality $f \leq B$ for some fixed $V \in \Phi, V \subset \Omega$. In view of Proposition 3, it is sufficient to show that, for every $y \in \Omega, f$ is bounded from above on some neighborhood of $y$. Let $y$ be an arbitrary element of $\Omega$. By the hypothesis of root-approximability, $y$ has a sequence of successive square roots $y^{1 / 2^{n}}$, with $y^{1 / 2^{n}} \longrightarrow e$. There exists an integer $N$ such that $y y^{1 / 2^{N}} \in \Omega$. We can take a chain $\left(V_{j}\right)_{1 \leq j \leq 2^{N}+1}$ of symmetric open members of $\Phi$ with

$$
V_{2^{N}+1} \subset V_{2^{N}} \subset \cdots \subset V_{2} \subset V_{1}
$$

and the properties:

$$
\begin{aligned}
& \text { (i) } V_{1}^{2^{N}+1} \subset V ; \\
& \text { (ii) } y^{-1 / 2^{N}} V_{j} \subset V_{j-1} y^{-1 / 2^{N}} \cap y^{-1 / 2^{N}} V_{j-1} .
\end{aligned}
$$

We shall prove that on the neighborhood $V_{2^{N}+1} y$ of $y$ the function $f$ satisfies an inequality of the form $f \leq C$, for some constant $C$

$$
C=\left(1-\frac{2^{N}}{2^{N}+1}\right) B+\frac{2^{N}}{2^{N}+1} f\left(y y^{1 / 2^{N}}\right),
$$

depending on $B, y$ and $N$. Given $x \in V_{2^{N}+1}$, define $X=\left(y^{-1 / 2^{N}} x\right)^{2^{N}}$. The property (ii) above shows that there exists $x_{1} \in V_{2^{N}}$ such that

$$
y^{-1 / 2^{N}} x=x_{1} y^{-1 / 2^{N}} \text {. }
$$

Thus,

$$
\begin{gathered}
X=\left(x_{1} y^{-1 / 2^{N}}\right) \cdots\left(x_{1} y^{-1 / 2^{N}}\right) \\
=x_{1}\left(y^{-1 / 2^{N}} x_{1}\right) \cdots\left(y^{-1 / 2^{N}} x_{1}\right) y^{-1 / 2^{N}} \\
=x_{1}\left(y^{-1 / 2^{N}} x_{1}\right)^{2^{N}-1} y^{-1 / 2^{N}} .
\end{gathered}
$$

This process will enable us to construct, for $1 \leq j \leq 2^{N}$, suitable $x_{j} \in V_{2^{N}+1-j}$ satisfying

$$
X=x_{1} \cdots x_{j}\left(y^{-1 / 2^{N}} x_{j}\right)^{2^{N}-j} y^{-j / 2^{N}} .
$$

For $j=2^{N}$, we have

$$
X=x_{1} \cdots x_{2^{N}} y^{-1} ;
$$

therefore $X y \in V_{1}^{2^{N}}$ and by the property $(i)$

$$
x X y \in V_{1}^{2^{N}+1} \subset V .
$$


By Lemma 1, we have

$$
\begin{aligned}
f(x y) & \leq\left(1-\frac{2^{N}}{2^{N}+1}\right) f(x X y)+\frac{2^{N}}{2^{N}+1} f\left(y y^{1 / 2^{N}}\right) \\
& \leq\left(1-\frac{2^{N}}{2^{N}+1}\right) B+\frac{2^{N}}{2^{N}+1} f\left(y y^{1 / 2^{N}}\right) .
\end{aligned}
$$

The proof is complete.

\section{Midconvex hull And Ostrowski's theorem}

Lemma 2. Let $\Omega$ be an open midconvex set in a root-approximable group $G, f$ : $\Omega \longrightarrow \mathbf{R}$ a globally midconvex function. Suppose that $E \subset \Omega$, and $f \leq C$ on $E$. Then $f \leq C$ on $H(E) \cap \Omega$.

Proof. We can express an element $x$ of $H(E)$ as

$$
x=x_{1} a_{n_{1}}^{m_{1}} a_{n_{2}}^{m_{2}} \cdots a_{n_{k-1}}^{m_{k-1}}
$$

with suitable $x_{1} \in E, a_{n_{j}} \in G, x_{2}=x_{1} a_{n_{1}}^{2_{1}} \in E, \cdots$, and generally

$$
x_{1} a_{n_{1}}^{m_{1}} a_{n_{2}}^{m_{2}} \cdots a_{n_{j-1}}^{2^{n_{j-1}}}=x_{j} \in E .
$$

An application of Lemma 1 gives the following inequality:

$$
f(x) \leq \alpha_{1} f\left(x_{1}\right)+\cdots+\alpha_{k} f\left(x_{k}\right)
$$

where $\alpha_{j}$ are calculated and checked to be of the form adapted (i.e. in binomial finite development), and

$$
\alpha_{1}+\cdots+\alpha_{k}=1
$$

We omit the details of this calculation. Therefore $f(x) \leq \alpha_{1} C+\cdots+\alpha_{k} C=C$.

The proof is complete.

Remark. Lemma 2 contains much more than what we need to prove Ostrowski's theorem. Indeed, it is well-known that in a locally compact group $G$ with the left Haar measure $\mu$ any set of the form $E^{-1} F$ (resp. of the form $E F$ ) with measurable sets $E$ and $F$ having positive measures $\mu(E)>0$ and $\mu(F)>0$ contains a nonempty $V \in \Phi$ (resp. a nonempty $V$ ). It follows that $\left(E^{-1} E\right)^{1 / 2}$ contains an open $W \in \Phi$, and $(E E)^{1 / 2}$ has a non empty interior $W$. One obtains

$$
W \subset H_{1}(E)=(E E)^{1 / 2},
$$

in the abelian case. Applying now the generalized Bernstein-Doetsch theorem as stated in our Theorem 1, we are in a position to complete the proof of our main result:

Theorem 2. Let $\Omega$ be an open midconvex set in a root-approximable locally compact non discrete abelian group $G, \mu$ a Haar measure on $G$ and $f: \Omega \longrightarrow \mathbf{R} a$ globally midconvex function. If $f$ is bounded from above on a set $E \subset \Omega$ with positive measure $\mu(E)>0$, then $f$ must be continuous everywhere on $\Omega$. 


\section{ACKNOWLEDGMENT}

We are in debt to the referee for his careful suggestions. We express our deep appreciation for interest, communication and conversations with Professors Gustave Choquet, Jean Pierre Demailly, Alain Dufresnoy, Bahman Mehri and Darioush Shademan. The final version of this work has been written when the first author was on sabbatical leave from the University of Tehran. We have to thank the University of Tehran and the Institut Fourier, University of Grenoble 1 ( Université Joseph Fourier) for their support and warm hospitality.

\section{REFERENCES}

[1] F. Bernstein and G. Doetsch, Zur Theorie der Konvexen Funktionen, Math. Ann. 76 (1915), 514-526.

[2] H. Blumberg, On convex functions, Trans. Amer. Math. Soc. 20 (1919), 40-44.

[3] N. Bourbaki, Topologie générale, chap. 3 et 4, 2-ième éd. (Actualités Scientifiques et Industrielles no. 1143), Hermann, Paris, (1960). MR 25:4021

[4] N. Bourbaki, Espaces vectoriels topologiques, chap. 1 et 2, 2-ième éd. (Actualités Scientifiques et Industrielles no. 1198), Hermann, Paris, (1966).

[5] A. Chademan, Sur les notions élémentaires de la théorie spectrale, Thèse de Doctorat 3-ième cycle, Univ. Paris VI, 1970.

[6] A. Chademan and F. Mirzapour, Boundedness properties of midconvex functions in locally comact groups, (in Proc. of the 26-th AIMC, March 1995, published by the Iranian Math. Society and University of Kerman, Kerman, Iran), 1995, 59-63. CMP 98:07

[7] R. Ger, Some remarks on convex functions, Fund. Math. 66(1970), 255-262. MR 40:7405

[8] R. Ger, n-convex functions in linear spaces, Aequationes Math. 11 (1974), 172-176. MR 50:10584

[9] R. Ger and M. Kuczma, On the boundedness and continuity of convex functions and additive functions, Aequationes Math. 4(1970), 157-162. MR 41:8605

[10] I.I. Hirschman and D.V. Widder, The convolution transform, Princeton University Press, Princeton, New Jersey, (1955). MR 17:479c

[11] J. L. W. V. Jensen, Sur les fonctions convexes et les inégalités entre les valeurs moyennes, Acta Math. 30(1906), 175-193.

[12] Z. Kominek, On additive and convex functionals, Rad. Mat. 3 (1987), 267-279. MR 89e:26029

[13] Z. Kominek and M. Kuczma,, Theorems of Bernstein-Doetsch, Piccard and Mehdi and semilinear topology, Arch. Math. 52 (1989), 595-602. MR 90i:46017

[14] M. Kuczma, An introduction to the theory of functional equations and inequations. Cauchy's equation and Jensen's inequality, PWN i Uniwersytest Slcaski, Warszawa, (1985). MR 86i:39008

[15] M.R. Mehdi, On convex functions, J. Lond. Math. Soc. 39 (1964), 321-326. MR 28:5153

[16] C.T. Ng and K. Nikodem, On approximately convex functions, Proc. Amer. Math. Soc. 118 (1993), 103-108. MR 93f:26006

[17] A. Ostrowski, Uber die Funktionalgleichung der Exponentialfunktionen und verwande Funktionalgleichungen, Jahresber. Deut. Math. Ver., 38(1929), 54-62.

[18] A.W. Roberts and D.A. Varberg, Convex functions, Academic Press, New York, (1973). MR 56:1201

[19] W. Sierpinski, Sur les fonctions convexes mesurables; Fund. Math. 1 (1920), 125-129.

[20] A. Weil, L' intégration dans les groupes topologiques et ses applications, 2-ième éd., (Actualités Scientifiques et Industrielles no. 1145), Hermann, Paris, (1965).

Department of Mathematics and Computer Science, Faculty of Science, University of Tehran, Tehran, Iran

E-mail address: chademan@khayam.ut.ac.ir

Department of Mathematics, University of Zanjan, Zanjan, Iran 